\title{
Measurement of matter-antimatter differences in beauty baryon decays
}

\author{
The LHCb collaboration ${ }^{\dagger}$
}

\begin{abstract}
Differences in the behaviour of matter and antimatter have been observed in $K$ and $B$ meson decays, but not yet in any baryon decay. Such differences are associated with the non-invariance of fundamental interactions under the combined chargeconjugation and parity transformations, known as CP violation. Here, using data from the LHCb experiment at the Large Hadron Collider, we search for CP-violating asymmetries in the decay angle distributions of $\Lambda_{b}^{0}$ baryons decaying to $p \pi^{-} \pi^{+} \pi^{-}$and $p \pi^{-} K^{+} K^{-}$final states. These four-body hadronic decays are a promising place to search for sources of $C P$ violation both within and beyond the standard model of particle physics. We find evidence for CP violation in $\Lambda_{b}^{0}$ to $p \pi^{-} \pi^{+} \pi^{-}$decays with a statistical significance corresponding to $\mathbf{3 . 3}$ standard deviations including systematic uncertainties. This represents the first evidence for $C P$ violation in the baryon sector.
\end{abstract}

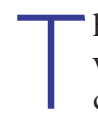
he asymmetry between matter and antimatter is related to the violation of the $C P$ symmetry (CPV), where $C$ and $P$ are the charge-conjugation and parity operators. $C P$ violation is accommodated in the standard model (SM) of particle physics by the Cabibbo-Kobayashi-Maskawa (CKM) mechanism that describes the transitions between up- and down-type quarks ${ }^{1,2}$, in which quark decays proceed by the emission of a virtual $W$ boson and where the phases of the couplings change sign between quarks and antiquarks. However, the amount of CPV predicted by the CKM mechanism is not sufficient to explain our matter-dominated Universe $\mathrm{e}^{3,4}$ and other sources of CPV are expected to exist. The initial discovery of CPV was in neutral $K$ meson decays ${ }^{5}$, and more recently it has been observed in $B^{0}$ (refs 6,7), $B^{+}$(refs 8-11), and $B_{s}^{0}$ (ref. 12) meson decays, but it has never been observed in the decays of any baryon. Decays of the $\Lambda_{b}^{0}$ (bud) baryon to final states consisting of hadrons with no charm quarks are predicted to have non-negligible $C P$ asymmetries in the SM, as large as $20 \%$ for certain three-body decay modes $^{13}$. It is important to measure the size and nature of these $C P$ asymmetries in as many decay modes as possible, to determine whether they are consistent with the CKM mechanism or, if not, what extensions to the SM would be required to explain them ${ }^{14-16}$.

The decay processes studied in this article, $\Lambda_{b}^{0} \rightarrow p \pi^{-} \pi^{+} \pi^{-}$ and $\Lambda_{b}^{0} \rightarrow p \pi^{-} K^{+} K^{-}$, are mediated by the weak interaction and governed mainly by two amplitudes, expected to be of similar magnitude, from different diagrams describing quark-level $b \rightarrow$ $u \bar{u} d$ transitions, as shown in Fig. 1. Throughout this paper the inclusion of charge-conjugate reactions is implied, unless otherwise indicated. CPV could arise from the interference of two amplitudes with relative phases that differ between particle and antiparticle decays, leading to differences in the $\Lambda_{b}^{0}$ and $\bar{\Lambda}_{b}^{0}$ decay rates. The main source of this effect in the SM would be the large relative phase (referred to as $\alpha$ in the literature) between the product of the CKM matrix elements $V_{u b} V_{u d}^{*}$ and $V_{t b} V_{t d}^{*}$, which are present in the different diagrams depicted in Fig. 1. Parity violation (PV) is also expected in weak interactions, but has never been observed in $\Lambda_{b}^{0}$ decays.

To search for $C P$-violating effects one needs to measure $C P$ odd observables, which can be done by studying asymmetries in the $\widehat{T}$ operator. This is a unitary operator that reverses both the momentum and spin three-vectors ${ }^{17,18}$, and is different from the antiunitary time-reversal operator $T^{19,20}$ that also exchanges initial and final states. A non-zero $C P$-odd observable implies $C P$ violation, and similar considerations apply to $P$-odd observables and parity violation ${ }^{21}$. Furthermore, different values of $P$-odd observables for a decay and its charge conjugate would imply CPV. In this paper, scalar triple products of final-state particle momenta in the $\Lambda_{b}^{0}$ centre-of-mass frame are studied to search for $P$ - and $C P$ violating effects in four-body decays. These are defined as $C_{\widehat{T}}=\mathbf{p}_{p}$. $\left(\mathbf{p}_{h_{1}^{-}} \times \mathbf{p}_{h_{2}^{+}}\right)$for $\Lambda_{b}^{0}$ and $\bar{C}_{\widehat{T}}=\mathbf{p}_{\bar{p}} \cdot\left(\mathbf{p}_{h_{1}^{+}} \times \mathbf{p}_{h_{2}^{-}}\right)$for $\bar{\Lambda}_{b}^{0}$, where $h_{1}$ and $h_{2}$ are final-state hadrons: $h_{1}=\pi$ and $h_{2}=K$ for $\Lambda_{b}^{0} \rightarrow p \pi^{-} K^{+} K^{-}$ and $h_{1}=h_{2}=\pi$ for $\Lambda_{b}^{0} \rightarrow p \pi^{-} \pi^{+} \pi^{-}$. In the latter case there is an inherent ambiguity in the choice of the pion for $h_{1}$ that is resolved by taking that with the larger momentum in the $\Lambda_{b}^{0}$ rest frame, referred to as $\pi_{\text {fast }}$. The following asymmetries may then be defined ${ }^{22,23}$ :

$$
\begin{gathered}
A_{\widehat{T}}\left(C_{\widehat{T}}\right)=\frac{N\left(C_{\widehat{T}}>0\right)-N\left(C_{\widehat{T}}<0\right)}{N\left(C_{\widehat{T}}>0\right)+N\left(C_{\widehat{T}}<0\right)} \\
\bar{A}_{\widehat{T}}\left(\bar{C}_{\widehat{T}}\right)=\frac{\bar{N}\left(-\bar{C}_{\widehat{T}}>0\right)-\bar{N}\left(-\bar{C}_{\widehat{T}}<0\right)}{\bar{N}\left(-\bar{C}_{\widehat{T}}>0\right)+\bar{N}\left(-\bar{C}_{\widehat{T}}<0\right)}
\end{gathered}
$$

where $N$ and $\bar{N}$ are the numbers of $\Lambda_{b}^{0}$ and $\bar{\Lambda}_{b}^{0}$ decays. These asymmetries are $P$-odd and $\widehat{T}$-odd and so change sign under $P$ or $\widehat{T}$ transformations, that is, $A_{\widehat{T}}\left(C_{\widehat{T}}\right)=-A_{\widehat{T}}\left(-C_{\widehat{T}}\right)$ or $\bar{A}_{\widehat{T}}\left(\bar{C}_{\widehat{T}}\right)=$ $-\bar{A}_{\widehat{T}}\left(-\bar{C}_{\widehat{T}}\right)$. The $P$ - and $C P$-violating observables are defined as

$$
a_{P}^{\widehat{T} \text {-odd }}=\frac{1}{2}\left(A_{\widehat{T}}+\bar{A}_{\widehat{T}}\right), \quad a_{C P}^{\widehat{T} \text {-odd }}=\frac{1}{2}\left(A_{\widehat{T}}-\bar{A}_{\widehat{T}}\right)
$$

and a significant deviation from zero would signal PV or $\mathrm{CPV}$, respectively.

Searches for CPV with triple-product asymmetries are particularly suited to $\Lambda_{b}^{0}$ four-body decays to hadrons with no charm quark ${ }^{24}$ thanks to the rich resonant substructure, dominated by $\Delta(1232)^{++} \rightarrow p \pi^{+}$and $\rho(770)^{0} \rightarrow \pi^{+} \pi^{-}$resonances in the $\Lambda_{b}^{0} \rightarrow p \pi^{-} \pi^{+} \pi^{-}$final state. The observable $a_{C P}^{\widehat{T} \text {-odd }}$ is sensitive to the interference of $\widehat{T}$-even and $\widehat{T}$-odd amplitudes with different $C P$-odd ('weak') phases. Unlike the overall asymmetry in the decay rate that is sensitive to the interference of $\widehat{T}$-even amplitudes, $a_{C P}^{\widehat{T} \text {-odd }}$ does not require a non-vanishing difference 

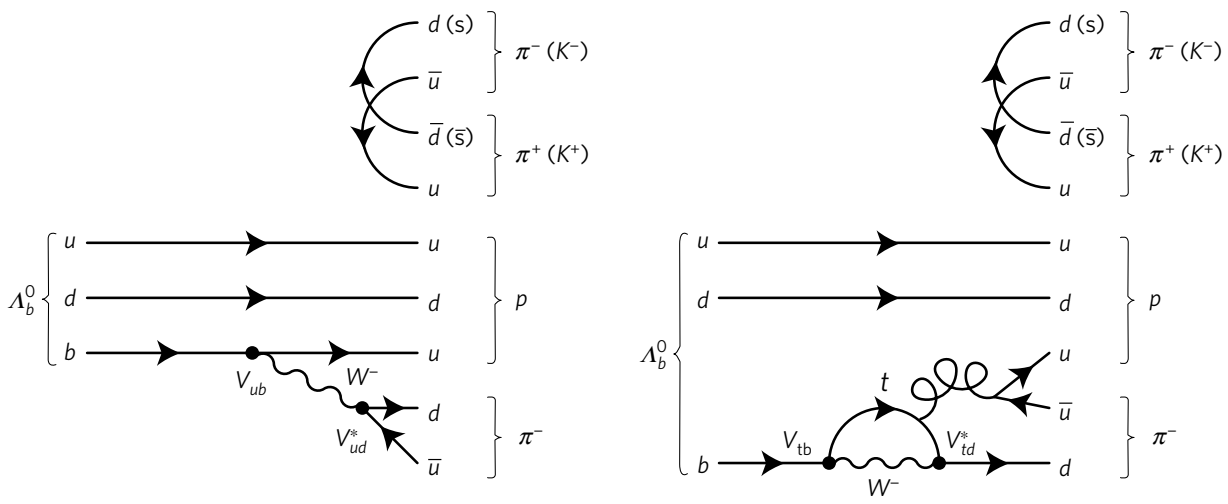

Figure 1 | Dominant Feynman diagrams for $\Lambda_{b}^{0} \rightarrow p \pi^{-} \pi^{+} \pi^{-}$and $\Lambda_{b}^{0} \rightarrow p \pi^{-} K^{+} K^{-}$transitions. The two diagrams show the transitions that contribute most strongly to $\Lambda_{b}^{0} \rightarrow p \pi^{-} \pi^{+} \pi^{-}$and $\Lambda_{b}^{0} \rightarrow p \pi^{-} K^{+} K^{-}$decays. In both cases, a pair of $\pi^{+} \pi^{-}\left(K^{+} K^{-}\right)$is produced by gluon emission from the light quarks $(u, d)$. The difference is in the $b$ quark decay that happens on the left through a virtual $W^{-}$boson emission ('tree diagram') and on the right as a virtual $W^{-}$boson emission and absorption together with a gluon emission ('loop diagram'). The magnitudes of the two amplitudes are expected to be comparable, and each is proportional to the product of the CKM matrix elements involved, which are shown in the figure.
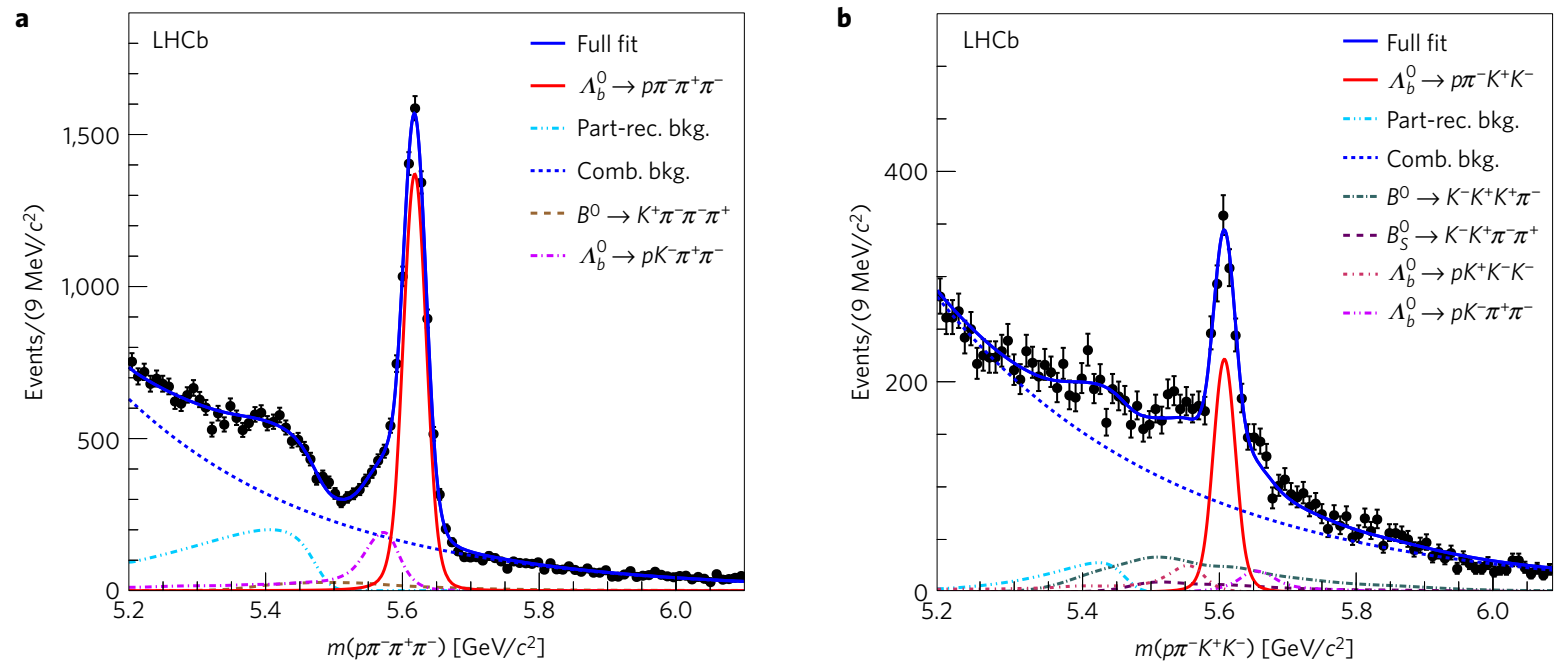

Figure 2 | Reconstructed invariant mass fits used to extract the signal yields. The invariant mass distributions for (a) $\Lambda_{b}^{0} \rightarrow p \pi^{-} \pi^{+} \pi^{-}$and $(\mathbf{b})$ $\Lambda_{b}^{0} \rightarrow p \pi^{-} K^{+} K^{-}$decays are shown. A fit is overlaid on top of the data points, with solid and dotted lines describing the projections of the fit results for each of the components described in the text and listed in the legend. Uncertainties on the data points are statistical only and represent one standard deviations, calculated assuming Poisson-distributed entries.

in the $C P$-invariant ('strong') phase between the contributing amplitudes $^{19,25}$. The observables $A_{\widehat{T}}, \bar{A}_{\widehat{T}}, a_{P}^{\widehat{T} \text {-odd }}$ and $a_{C P}^{\widehat{T} \text {-odd }}$ are, by construction, largely insensitive to particle-antiparticle production asymmetries and detector-induced charge asymmetries ${ }^{26}$.

This article describes measurements of the $C P$ - and $P$-violating asymmetries introduced in equation (3) in $\Lambda_{b}^{0} \rightarrow p \pi^{-} \pi^{+} \pi^{-}$and $\Lambda_{b}^{0} \rightarrow p \pi^{-} K^{+} K^{-}$decays. The asymmetries are measured first for the entire phase space of the decay, integrating over all possible final-state configurations, and then in different regions of phase space so as to enhance sensitivity to localized CPV. The analysis is performed using proton-proton collision data collected by the $\mathrm{LHCb}$ detector, corresponding to $3.0 \mathrm{fb}^{-1}$ of integrated luminosity at centre-of-mass energies of 7 and $8 \mathrm{TeV}$, and exploits the copious production of $\Lambda_{b}^{0}$ baryons at the LHC, which constitutes around $20 \%$ of all $b$ hadrons produced ${ }^{27}$. Control samples of $\Lambda_{b}^{0} \rightarrow p K^{-} \pi^{+} \pi^{-}$ and $\Lambda_{b}^{0} \rightarrow \Lambda_{c}^{+} \pi^{-}$decays, with $\Lambda_{c}^{+}$decaying to $p K^{-} \pi^{+}, p \pi^{-} \pi^{+}$, and $p K^{-} K^{+}$final states, are used to optimize the event selection and study systematic effects; the most abundant control sample consists of $\Lambda_{b}^{0} \rightarrow \Lambda_{c}^{+}\left(p K^{-} \pi^{+}\right) \pi^{-}$decays mediated by $b \rightarrow c$ quark transitions in which no CPV is expected ${ }^{28}$. To avoid introducing biases in the results, all aspects of the analysis, including the selection, phase space regions, and procedure used to determine the statistical significance of the results, were fixed before the data were examined.

The LHCb detector ${ }^{29,30}$ is designed to collect data of $b$-hadron decays produced from proton-proton collisions at the Large Hadron Collider. It instruments a region around the proton beam axis, covering the polar angles between 10 and $250 \mathrm{mrad}$, where approximately $24 \%$ of the $b$-hadron decays occur ${ }^{31}$. The detector includes a high-precision tracking system with a dipole magnet, providing measurements of the momentum and decay vertex position of particle decays. Different types of charged particles are distinguished using information from two ring-imaging Cherenkov detectors, a calorimeter and a muon system. Simulated samples of $\Lambda_{b}^{0}$ signal modes and control samples are used in this analysis to verify the experimental method and to study certain systematic effects. These simulated events model the experimental conditions in detail, including the proton-proton collision, the decays of the particles, and the response of the detector. The software used is described in refs $32-38$. The online event selection is performed by a trigger system that takes fast decisions about which events to record. It consists of a hardware stage, based on information from the 
Table 1 | Definition of binning scheme $A$ for the decay mode $\Lambda_{b}^{0} \rightarrow p \pi^{-} \pi^{+} \pi^{-}$.

\begin{tabular}{|c|c|c|c|c|}
\hline Phase space bin & $m\left(p \pi^{+}\right)$ & $m\left(p \pi_{\text {slow }}^{-}\right)$ & $m\left(\pi^{+} \pi_{\text {slow }}^{-}\right), m\left(\pi^{+} \pi_{\text {fast }}^{-}\right)$ & $|\Phi|$ \\
\hline 1 & $(1.07,1.23)$ & & & $\left(0, \frac{\pi}{2}\right)$ \\
\hline 2 & $(1.07,1.23)$ & & & $\left(\frac{\pi}{2}, \pi\right)$ \\
\hline 3 & $(1.23,1.35)$ & & & $\left(0, \frac{\pi}{2}\right)$ \\
\hline 4 & $(1.23,1.35)$ & & & $\left(\frac{\pi}{2}, \pi\right)$ \\
\hline 5 & $(1.35,5.34)$ & $(1.07,2.00)$ & $m\left(\pi^{+} \pi_{\text {slow }}^{-}\right)<0.78$ or $m\left(\pi^{+} \pi_{\text {fast }}^{-}\right)<0.78$ & $\left(0, \frac{\pi}{2}\right)$ \\
\hline 6 & $(1.35,5.34)$ & $(1.07,2.00)$ & $m\left(\pi^{+} \pi_{\text {slow }}^{-}\right)<0.78$ or $m\left(\pi^{+} \pi_{\text {fast }}^{-}\right)<0.78$ & $\left(\frac{\pi}{2}, \pi\right)$ \\
\hline 7 & $(1.35,5.34)$ & $(1.07,2.00)$ & $m\left(\pi^{+} \pi_{\text {slow }}^{-}\right)>0.78$ and $m\left(\pi^{+} \pi_{\text {fast }}^{-}\right)>0.78$ & $\left(0, \frac{\pi}{2}\right)$ \\
\hline 8 & $(1.35,5.34)$ & $(1.07,2.00)$ & $m\left(\pi^{+} \pi_{\text {slow }}^{-}\right)>0.78$ and $m\left(\pi^{+} \pi_{\text {fast }}^{-}\right)>0.78$ & $\left(\frac{\pi}{2}, \pi\right)$ \\
\hline 9 & $(1.35,5.34)$ & $(2.00,4.00)$ & $m\left(\pi^{+} \pi_{\text {slow }}^{-}\right)<0.78$ or $m\left(\pi^{+} \pi_{\text {fast }}^{-}\right)<0.78$ & $\left(0, \frac{\pi}{2}\right)$ \\
\hline 10 & $(1.35,5.34)$ & $(2.00,4.00)$ & $m\left(\pi^{+} \pi_{\text {slow }}^{-}\right)<0.78$ or $m\left(\pi^{+} \pi_{\text {fast }}^{-}\right)<0.78$ & $\left(\frac{\pi}{2}, \pi\right)$ \\
\hline 11 & $(1.35,5.34)$ & $(2.00,4.00)$ & $m\left(\pi^{+} \pi_{\text {slow }}^{-}\right)>0.78$ and $m\left(\pi^{+} \pi_{\text {fast }}^{-}\right)>0.78$ & $\left(0, \frac{\pi}{2}\right)$ \\
\hline 12 & $(1.35,5.34)$ & $(2.00,4.00)$ & $m\left(\pi^{+} \pi_{\text {slow }}^{-}\right)>0.78$ and $m\left(\pi^{+} \pi_{\text {fast }}^{-}\right)>0.78$ & $\left(\frac{\pi}{2}, \pi\right)$ \\
\hline
\end{tabular}

Binning scheme $\mathrm{A}$ is defined to exploit interference patterns arising from the resonant structure of the decay. Bins $1-4$ focus on the region dominated by the $\Delta(1232)^{++} \rightarrow p \pi^{+}$resonance. The other eight bins are defined to study regions where $p \pi^{-}$resonances are present (5-8) on either side of the $\rho(770)^{0} \rightarrow \pi^{+} \pi^{-}$resonances (5-12). Further splitting for $|\Phi|$ lower or greater than $\pi / 2$ is done to reduce potential dilution of asymmetries, as suggested in ref. 19. Masses are in units of $\mathrm{GeV} / \mathrm{c}^{2}$.

calorimeter and muon systems, followed by a software stage, which applies a full event reconstruction. The software trigger requires $\Lambda_{b}^{0}$ candidates to be consistent with a $b$-hadron decay topology, with tracks originating from a secondary vertex detached from the primary $p p$ collision point. The mean $\Lambda_{b}^{0}$ lifetime is 1.5 ps (ref. 39), which corresponds to a typical flight distance of a few millimetres in the LHCb.

The $\Lambda_{b}^{0} \rightarrow p \pi^{-} h^{+} h^{-}$candidates are formed by combining tracks identified as protons, pions, or kaons that originate from a common vertex. The proton or antiproton identifies the candidate as a $\Lambda_{b}^{0}$ or $\bar{\Lambda}_{b}^{0}$. There are backgrounds from $b$-hadron decays to charm hadrons that are suppressed by reconstructing the appropriate two- or three-body invariant masses, and requiring them to differ from the known charm hadron masses by at least three times the experimental resolution. For the $\Lambda_{b}^{0} \rightarrow \Lambda_{c}^{+} \pi^{-}$control mode, only the $\Lambda_{b}^{0} \rightarrow p h^{+} h^{-} \pi^{-}$events with reconstructed $p h^{+} h^{-}$invariant mass between 2.23 and $2.31 \mathrm{GeV} / c^{2}$ are retained.

A boosted decision tree (BDT) classifier ${ }^{40}$ is constructed from a set of kinematic variables that discriminate between signal and background. The signal and background training samples used for the BDT are derived from the $\Lambda_{b}^{0} \rightarrow p K^{-} \pi^{+} \pi^{-}$control sample, since its kinematics and topology are similar to the decays under study; background in this sample is subtracted with the sPlot technique ${ }^{41}$, a statistical technique to disentangle signal and background contributions. The background training sample consists of candidates that lie far from the signal mass peak, between 5.85 and $6.40 \mathrm{GeV} / c^{2}$. The control modes $\Lambda_{b}^{0} \rightarrow \Lambda_{c}^{+}\left(p \pi^{+} \pi^{-}\right) \pi^{-}$ and $\Lambda_{b}^{0} \rightarrow \Lambda_{c}^{+}\left(p K^{-} K^{+}\right) \pi^{-}$are used to optimize the particle identification criteria for the signal mode with the same final state. For events in which multiple candidates pass all selection criteria for a given mode, one candidate is retained at random and the rest discarded.

Unbinned extended maximum likelihood fits to the $p \pi^{-} \pi^{+} \pi^{-}$ and the $p \pi^{-} K^{+} K^{-}$invariant mass distributions are shown in Fig. 2. The invariant mass distribution of the $\Lambda_{b}^{0}$ signal is modelled by a Gaussian core with power-law tails ${ }^{42}$, with the mean and the width of the Gaussian determined from the fit to data. The combinatorial background is modelled by an exponential distribution with the rate parameter extracted from data. All other parameters of the fit model are taken from simulations except the yields. Partially reconstructed $\Lambda_{b}^{0}$ decays are described by an empirical function ${ }^{43}$ convolved with a Gaussian function to account for resolution effects. The shapes of backgrounds from other $b$-hadron decays due to incorrectly identified particles, for example, kaons identified as pions or protons identified as kaons, are modelled using simulated events. These consist mainly of $\Lambda_{b}^{0} \rightarrow p K^{-} \pi^{+} \pi^{-}$and $B^{0} \rightarrow K^{+} \pi^{-} \pi^{-} \pi^{+}$decays for the $\Lambda_{b}^{0} \rightarrow p \pi^{-} \pi^{+} \pi^{-}$sample and of similar final states for the $\Lambda_{b}^{0} \rightarrow p \pi^{-} K^{+} K^{-}$sample, as shown in Fig. 2. The yields of these contributions are obtained from fits to data reconstructed under the appropriate mass hypotheses for the final-state particles. The signal yields of $\Lambda_{b}^{0} \rightarrow p \pi^{-} \pi^{+} \pi^{-}$and $\Lambda_{b}^{0} \rightarrow p \pi^{-} K^{+} K^{-}$are $6,646 \pm 105$ and $1,030 \pm 56$, respectively. This is the first observation of these decay modes.

Signal candidates are split into four categories according to $\Lambda_{b}^{0}$ or $\bar{\Lambda}_{b}^{0}$ flavour and the sign of $C_{\widehat{T}}$ or $\bar{C}_{\widehat{T}}$ to calculate the asymmetries defined in equations (1) and (2). The reconstruction efficiency for signal candidates with $C_{\widehat{T}}>0$ is identical to that with $C_{\widehat{T}}<0$ within the statistical uncertainties of the control sample, and likewise for $\bar{C}_{\widehat{T}}$, which indicates that the detector and the reconstruction program do not bias this measurement. This check is performed both on the $\Lambda_{b}^{0} \rightarrow \Lambda_{c}^{+}\left(p K^{-} \pi^{+}\right) \pi^{-}$data control sample and on large samples of simulated events, using yields about 30 times those found in data, which are generated with no $C P$ asymmetry. The $C P$ asymmetry measured in the control sample is $a_{C P}^{\widehat{T} \text {-odd }}\left(\Lambda_{c}^{+} \pi^{-}\right)=(0.15 \pm 0.31) \%$, compatible with $C P$ symmetry. The asymmetries $A_{\widehat{T}}$ and $\bar{A}_{\widehat{T}}$ in the signal samples are measured with a simultaneous unbinned maximum likelihood fit to the invariant mass distributions of the different signal categories, and are found to be uncorrelated. Corresponding asymmetries for each of the background components are also measured in the fit; they are found to be consistent with zero, and do not lead to significant systematic uncertainties in the signal asymmetries. The values of $a_{C P}^{\widehat{T} \text {-odd }}$ and $a_{P}^{\widehat{T} \text {-odd }}$ are then calculated from $A_{\widehat{T}}$ and $\bar{A}_{\widehat{T}}$.

In four-body particle decays, the $C P$ asymmetries may vary over

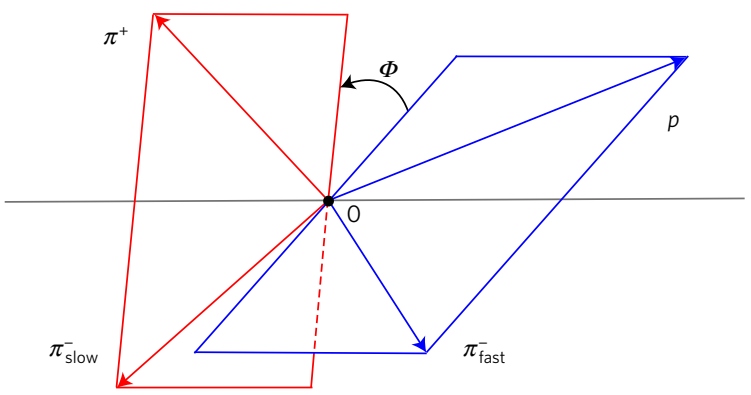

Figure 3 | Definition of the $\boldsymbol{\Phi}$ angle. The decay planes formed by the $p \pi_{\text {fast }}^{-}$ (blue) and the $\pi_{\text {slow }}^{-} \pi^{+}$(red) systems in the $\Lambda_{b}^{0}$ rest frame. The momenta of the particles, represented by vectors, determine the two decay planes and the angle $\Phi \in[-\pi, \pi]$ (ref. 19) measures their relative orientation. 

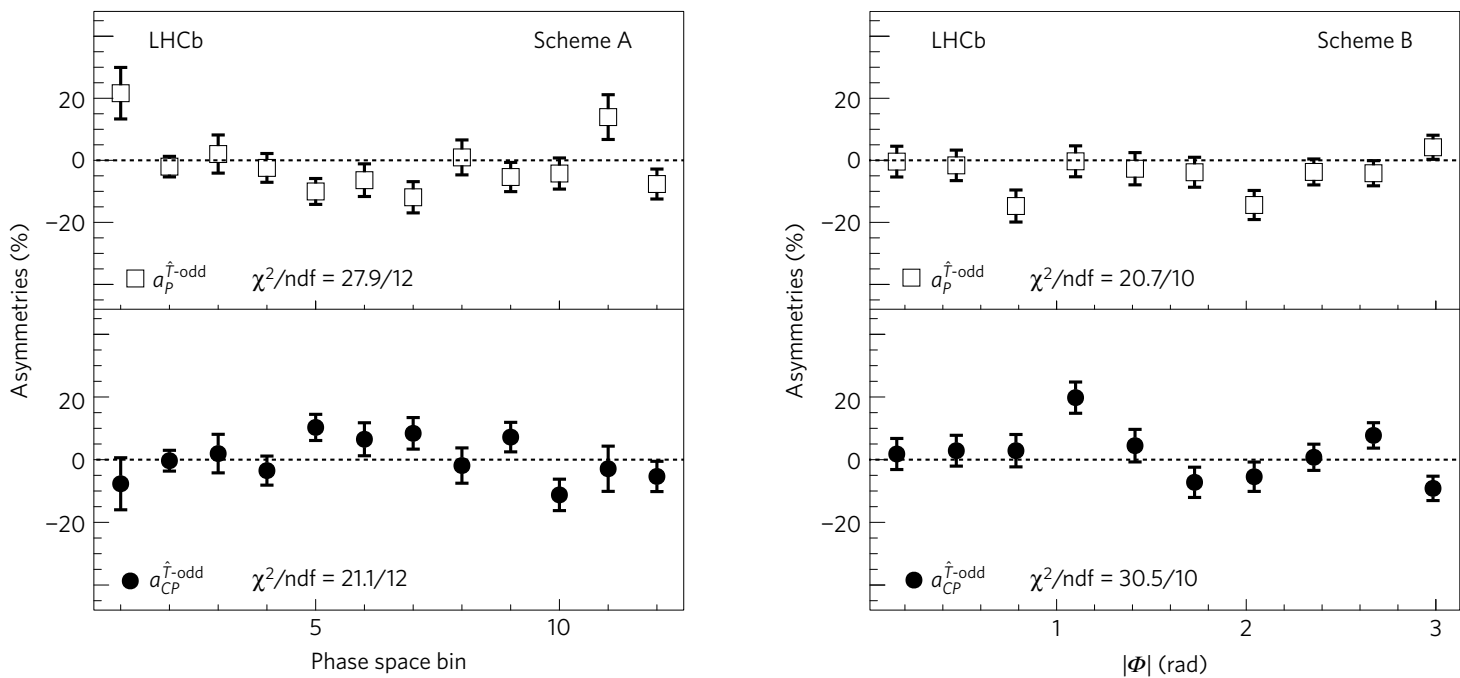

Figure 4 | Distributions of the asymmetries. The results of the fit in each region of binning schemes $A$ and $B$ are shown. The asymmetries $a_{p}^{\widehat{T}-\text { odd }}$ and $a_{C P}^{T-\text { odd }}$ for $\Lambda_{b}^{0} \rightarrow p \pi^{-} \pi^{+} \pi^{-}$decays are represented by open boxes and filled circles, respectively. The error bars represent one standard deviation, calculated as the sum in quadrature of the statistical uncertainty resulting from the fit to the invariant mass distribution and the systematic uncertainties estimated as described in the main text. The values of the $\chi^{2} /$ ndf are quoted for the $P$ - and $C P$-conserving hypotheses for each binning scheme, where ndf indicates the number of degrees of freedom.

the phase space due to resonant contributions or their interference effects, possibly cancelling when integrated over the whole phase space. Therefore, the asymmetries are measured in different regions of phase space for the $\Lambda_{b}^{0} \rightarrow p \pi^{-} \pi^{+} \pi^{-}$decay using two binning schemes, defined before examining the data. Scheme A, defined in Table 1, is designed to isolate regions of phase space according to their dominant resonant contributions. Scheme B exploits in more detail the interference of contributions which could be visible as a function of the angle $\Phi$ between the decay planes formed by the $p \pi_{\text {fast }}^{-}$and the $\pi_{\text {slow }}^{-} \pi^{+}$systems, as illustrated in Fig. 3. Scheme B has ten non-overlapping bins of width $\pi / 10$ in $|\Phi|$. For every bin in each of the schemes, the $\Lambda_{b}^{0}$ efficiencies for $C_{\widehat{T}}>0$ and $C_{\widehat{T}}<0$ are compared and found to be equal within uncertainties, and likewise the $\bar{\Lambda}_{b}^{0}$ efficiencies for $\bar{C}_{\widehat{T}}>0$ and $\bar{C}_{\widehat{T}}<0$. The analysis technique is validated on the $\Lambda_{b}^{0} \rightarrow \Lambda_{c}^{+}\left(p K^{-} \pi^{+}\right) \pi^{-}$control sample, for which the angle $\Phi$ is defined by the decay planes of the $p K^{-}$and $\pi^{+} \pi^{-}$ pairs, and on simulated signal events.

The asymmetries measured in $\Lambda_{b}^{0} \rightarrow p \pi^{-} \pi^{+} \pi^{-}$decays with these two binning schemes are shown in Fig. 4 and reported in Table 2, together with the integrated measurements. For each scheme individually, the compatibility with the $C P$-symmetry hypothesis is evaluated by means of a $\chi^{2}$ test, with $\chi^{2}=R^{T} V^{-1} R$, where $R$ is the array of $a_{C P}^{\widehat{T} \text {-odd }}$ measurements and $V$ is the covariance matrix, which is the sum of the statistical and systematic covariance matrices. An average systematic uncertainty, whose evaluation is discussed below, is assigned for all bins. The systematic uncertainties are assumed to be fully correlated; their contribution is small compared to the statistical uncertainties. The $p$-values of the $C P$ symmetry hypothesis are $4.9 \times 10^{-2}$ and $7.1 \times 10^{-4}$ for schemes $\mathrm{A}$ and $\mathrm{B}$, respectively, corresponding to statistical significances of 2.0 and 3.4 Gaussian standard deviations $(\sigma)$. A similar $\chi^{2}$ test is performed on $a_{p}^{\widehat{T} \text {-odd }}$ measurements with $p$-values for the $P$ symmetry hypothesis of $5.8 \times 10^{-3}(2.8 \sigma)$ and $2.4 \times 10^{-2}(2.3 \sigma)$, for scheme $\mathrm{A}$ and $\mathrm{B}$, respectively. The overall significance for $\mathrm{CPV}$ in $\Lambda_{b}^{0} \rightarrow p \pi^{-} \pi^{+} \pi^{-}$decays from the results of schemes $\mathrm{A}$ and $\mathrm{B}$ is determined by means of a permutation test ${ }^{44}$, taking into account correlations among the results. A sample of 40,000 pseudoexperiments is generated from the data by assigning each event a random $\Lambda_{b}^{0} / \bar{\Lambda}_{b}^{0}$ flavour such that $C P$ symmetry is enforced. The sign of $C_{\widehat{T}}$ is unchanged if a $\Lambda_{b}^{0}$ candidate stays $\Lambda_{b}^{0}$ and reversed if the $\Lambda_{b}^{0}$ candidate becomes $\bar{\Lambda}_{b}^{0}$. The $p$-value of the $C P$-symmetry hypothesis is determined as the fraction of pseudoexperiments with $\chi^{2}$ larger than that measured in data. Applying this method to the $\chi^{2}$ values from schemes $\mathrm{A}$ and $\mathrm{B}$ individually, the $p$-values obtained agree with those from the $\chi^{2}$ test within the uncertainty due to the limited number of pseudoexperiments. To assess a combined significance from the two schemes, the product of the two $p$-values measured in data is compared with the distribution of the product of the $p$-values of the two binning schemes from the pseudoexperiments. The fraction of pseudoexperiments whose $p$ value product is smaller than that seen in data determines the overall $p$-value of the combination of the two schemes ${ }^{45}$. An overall $p$-value of $9.8 \times 10^{-4}(3.3 \sigma)$ is obtained for the $C P$-symmetry hypothesis, including systematic uncertainties.

For the $\Lambda_{b}^{0} \rightarrow p \pi^{-} K^{+} K^{-}$decays, the smaller purity and signal yield of the sample do not permit PV and CPV to be probed with the same precision as for $\Lambda_{b}^{0} \rightarrow p \pi^{-} \pi^{+} \pi^{-}$, and therefore only two regions of phase space are considered. One spans $1.43<m\left(p K^{-}\right)<$ $2.00 \mathrm{GeV} / c^{2}$ (bin 1 ) and is dominated by excited $\Lambda$ resonances decaying to $p K$ and the other covers the remaining phase space, $2.00<m\left(p K^{-}\right)<4.99 \mathrm{GeV} / c^{2}$ (bin 2). The observables measured in these regions are given in Table 2 and are consistent with $C P$ and $P$ symmetry.

The main sources of systematic uncertainties for both $p \pi^{-} \pi^{+} \pi^{-}$ and $p \pi^{-} K^{+} K^{-}$decays are experimental effects that could introduce biases in the measured asymmetries. This is tested by measuring the asymmetry $a_{C P}^{\widehat{T} \text {-odd }}$, integrated over phase space and in various phase space regions, using the control sample $\Lambda_{b}^{0} \rightarrow \Lambda_{c}^{+}\left(p K^{-} \pi^{+}\right) \pi^{-}$, which is expected to exhibit negligible CPV. The results are in agreement with the $C P$-symmetry hypothesis; an uncertainty of $0.31 \%$ is assigned as a systematic uncertainty for the $a_{C P}^{\widehat{T} \text {-odd }}$ and $a_{P}^{\widehat{T} \text {-odd }}$ integrated measurements; an uncertainty of $0.60 \%$, the largest asymmetry from a fit to scheme B measurements using a range of efficiency and fit models, is assigned for the corresponding phase space measurements. The systematic uncertainty arising from the experimental resolution in the measurement of the triple products $C_{\widehat{T}}$ and $\bar{C}_{\widehat{T}}$, which could introduce a migration of events between the bins, is estimated from simulated samples of $\Lambda_{b}^{0} \rightarrow p \pi^{-} \pi^{+} \pi^{-}$and $\Lambda_{b}^{0} \rightarrow p \pi^{-} K^{+} K^{-}$decays where neither $P$ - nor $C P$-violating effects are present. The difference between the reconstructed and generated asymmetry is taken as a systematic uncertainty due to this effect, and is less than $0.06 \%$ in all cases. To assess the uncertainty associated 
Table 2 | Measurements of CP- and P-violating observables.

\begin{tabular}{|c|c|c|}
\hline & $a_{P}^{\widehat{T}}$-odd [\%] & $a_{C P}^{\widehat{T}}$-odd [\%] \\
\hline Scheme A & \multicolumn{2}{|c|}{$\Lambda_{b}^{0} \rightarrow p \pi^{-} \pi^{+} \pi^{-}$} \\
\hline 1 & $21.64 \pm 8.28 \pm 0.60$ & $-7.69 \pm 8.28 \pm 0.60$ \\
\hline 2 & $-2.04 \pm 3.26 \pm 0.60$ & $-0.33 \pm 3.26 \pm 0.60$ \\
\hline 3 & $2.03 \pm 6.12 \pm 0.60$ & $1.94 \pm 6.12 \pm 0.60$ \\
\hline 4 & $-2.45 \pm 4.60 \pm 0.60$ & $-3.49 \pm 4.60 \pm 0.60$ \\
\hline 5 & $-10.04 \pm 4.13 \pm 0.60$ & $10.29 \pm 4.13 \pm 0.60$ \\
\hline 6 & $-6.40 \pm 5.23 \pm 0.60$ & $6.51 \pm 5.23 \pm 0.60$ \\
\hline 7 & $-11.91 \pm 5.00 \pm 0.60$ & $8.40 \pm 5.00 \pm 0.60$ \\
\hline 8 & $0.94 \pm 5.60 \pm 0.60$ & $-1.88 \pm 5.60 \pm 0.60$ \\
\hline 9 & $-5.38 \pm 4.67 \pm 0.60$ & $7.20 \pm 4.67 \pm 0.60$ \\
\hline 10 & $-4.26 \pm 4.98 \pm 0.60$ & $-11.24 \pm 4.98 \pm 0.60$ \\
\hline 11 & $13.94 \pm 7.19 \pm 0.60$ & $-2.90 \pm 7.19 \pm 0.60$ \\
\hline 12 & $-7.64 \pm 4.79 \pm 0.60$ & $-5.35 \pm 4.79 \pm 0.60$ \\
\hline \multicolumn{3}{|l|}{ Scheme B } \\
\hline 1 & $-0.42 \pm 4.92 \pm 0.60$ & $1.81 \pm 4.92 \pm 0.60$ \\
\hline 2 & $-1.63 \pm 4.88 \pm 0.60$ & $2.86 \pm 4.88 \pm 0.60$ \\
\hline 3 & $-14.73 \pm 5.13 \pm 0.60$ & $2.87 \pm 5.13 \pm 0.60$ \\
\hline 4 & $-0.32 \pm 4.95 \pm 0.60$ & $19.79 \pm 4.95 \pm 0.60$ \\
\hline 5 & $-2.71 \pm 5.16 \pm 0.60$ & $4.47 \pm 5.16 \pm 0.60$ \\
\hline 6 & $-3.85 \pm 4.79 \pm 0.60$ & $-7.23 \pm 4.79 \pm 0.60$ \\
\hline 7 & $-14.40 \pm 4.65 \pm 0.60$ & $-5.44 \pm 4.65 \pm 0.60$ \\
\hline 8 & $-3.75 \pm 4.14 \pm 0.60$ & $0.76 \pm 4.14 \pm 0.60$ \\
\hline 9 & $-4.16 \pm 4.01 \pm 0.60$ & $7.74 \pm 4.01 \pm 0.60$ \\
\hline 10 & $4.21 \pm 3.84 \pm 0.60$ & $-9.16 \pm 3.84 \pm 0.60$ \\
\hline Integrated & $-3.71 \pm 1.45 \pm 0.32$ & $1.15 \pm 1.45 \pm 0.32$ \\
\hline Phase space bin & \multicolumn{2}{|c|}{$\Lambda_{b}^{0} \rightarrow p \pi^{-} K^{+} K^{-}$} \\
\hline 1 & $3.27 \pm 6.07 \pm 0.66$ & $-4.68 \pm 6.07 \pm 0.66$ \\
\hline$\underline{2}$ & $4.43 \pm 6.73 \pm 0.66$ & $4.73 \pm 6.73 \pm 0.66$ \\
\hline Integrated & $3.62 \pm 4.54 \pm 0.42$ & $-0.93 \pm 4.54 \pm 0.42$ \\
\hline \multicolumn{3}{|c|}{$\begin{array}{l}\text { The } C P \text { - and } P \text {-violating observables, }{ }_{C P}^{\hat{T}_{\text {-odd }}} \text { and } a_{P}^{\hat{T}_{-}^{-} \text {-odd }} \text {, resulting from the fit to the data are } \\
\text { listed with their statistical and systematic uncertainties. Each value is obtained through an } \\
\text { independent fit to a region of the phase space as described in the text and Table } 1 \text {. Results for } \\
\text { schemes } A \text { and } B \text { are outlined for } \Lambda_{b}^{\circ} \rightarrow p \pi^{-} \pi^{+} \pi^{-} \text {decays, and in two bins of phase space for } \\
\Lambda_{b}^{0} \rightarrow p \pi^{-} K^{+} K^{-} \text {decays, as defined in the text. The first column lists the bin number. For both } \\
\text { decay modes the measurement integrated over the phase space, performed independently, is } \\
\text { also shown. }\end{array}$} \\
\hline
\end{tabular}

with the fit models, alternative functions are used; these tests lead only to small changes in the asymmetries, the largest being $0.05 \%$. For $\Lambda_{b}^{0} \rightarrow p \pi^{-} K^{+} K^{-}$decays, this contribution is larger, about $0.28 \%$ for the $a_{C P}^{\widehat{T} \text {-odd }}$ and $a_{P}^{\widehat{T} \text {-odd }}$ asymmetries.

Further cross-checks are made to investigate the stability of the results with respect to different periods of recording data, different polarities of the spectrometer magnet, the choice made in the selection of multiple candidates, and the effect of the trigger and selection criteria. Alternative binning schemes are studied as a crosscheck, such as using 8 or 12 bins in $|\Phi|$ for $\Lambda_{b}^{0} \rightarrow p \pi^{-} \pi^{+} \pi^{-}$decays. For these alternative binning schemes, the significance of the CPV measurement of the modified scheme B is reduced to below $3 \sigma$. Nonetheless, the overall significance of the combination of these two additional binnings with schemes $\mathrm{A}$ and $\mathrm{B}$ remains above three standard deviations, with a $p$-value of $1.8 \times 10^{-3}(3.1 \sigma)$, consistent with the $3.3 \sigma$ result seen in the baseline analysis. An independent analysis of the data based on alternative selection criteria confirmed the results. It used a similar number of events, of which $73.4 \%$ are in common with the baseline analysis, and gave $p$-values for $C P$ symmetry of $3.4 \times 10^{-3}(2.9 \sigma)$ for scheme A and $1.4 \times 10^{-4}(3.8 \sigma)$ for scheme B.

In conclusion, a search for $P$ and $C P$ violation in $\Lambda_{b}^{0} \rightarrow p \pi^{-} \pi^{+} \pi^{-}$and $\Lambda_{b}^{0} \rightarrow p \pi^{-} K^{+} K^{-}$decays is performed on signal yields of $6,646 \pm 105$ and $1,030 \pm 56$ events. This is the first observation of these decay modes. Measurements of asymmetries in the entire phase space do not show any evidence of $P$ or $C P$ violation. Searches for localized $P$ or $C P$ violation are performed by measuring asymmetries in different regions of the phase space. The results are consistent with $C P$ symmetry for $\Lambda_{b}^{0} \rightarrow p \pi^{-} K^{+} K^{-}$decays, but evidence for $C P$ violation at the $3.3 \sigma$ level is found in $\Lambda_{b}^{0} \rightarrow p \pi^{-} \pi^{+} \pi^{-}$decays. No significant $P$ violation is found. This represents the first evidence of $C P$ violation in the baryon sector, and indicates an asymmetry between baryonic matter and antimatter.

Data availability. All data shown in histograms and plots are publicly available from HEPdata (https://hepdata.net).

Received 16 September 2016; accepted 21 December 2016; published online 30 January 2017

\section{References}

1. Cabibbo, N. Unitary symmetry and leptonic decays. Phys. Rev. Lett. 10, 531-533 (1963).

2. Kobayashi, M. \& Maskawa, T. CP violation in the renormalizable theory of weak interaction. Prog. Theor. Phys. 49, 652-657 (1973).

3. Sakharov, A. D. Violation of $C P$ invariance, $C$ asymmetry, and baryon asymmetry of the universe. JETP Lett. 5, 24-27 (1967); Sov. Phys. Usp. 34, 392-393 (1991).

4. Riotto, A. Theories of Baryogenesis. in In Proc. Summer School in High-energy Physics and Cosmology (eds Gava, E. et al.) 326-436 (World Scientific, 1998).

5. Christenson, J. H., Cronin, J. W., Fitch, V. L. \& Turlay, R. Evidence for the $2 \pi$ decay of the $K_{2}^{0}$ Meson. Phys. Rev. Lett. 13, 138-140 (1964).

6. Aubert, B. et al. Observation of $C P$ violation in the $B^{0}$ meson system. Phys. Rev. Lett. 87, 091801 (2001).

7. Abe, K. et al. Observation of large $C P$ violation in the neutral $B$ meson system. Phys. Rev. Lett. 87, 091802 (2001).

8. Aubert, B. et al. Improved measurement of the CKM angle $\gamma$ in $B^{\mp} \rightarrow D^{(*)} K^{(*) \mp}$ decays with a Dalitz plot analysis of $D$ decays to $K_{S}^{0} \pi^{+} \pi^{-}$and $K_{s}^{0} K^{+} K^{-}$. Phys. Rev. D 78, 034023 (2008).

9. Poluektov, A. et al. Evidence for direct $C P$ violation in the decay $B^{+} \rightarrow D^{(*)} K^{+}$, $D \rightarrow K^{+} \pi^{+} \pi^{-}$and measurement of the CKM phase $\phi_{3}$. Phys. Rev. D 81, 112002 (2010)

10. Aaij, R. et al. Observation of $C P$ violation in $B^{ \pm} \rightarrow D K^{ \pm}$decays. Phys. Lett. $B$ 712, 203-212 (2012); erratum 713, 351 (2012).

11. Aaij, R. et al. Measurement of $C P$ violation in the phase space of $B^{ \pm} \rightarrow K^{ \pm} \pi^{+} \pi^{-}$and $B^{ \pm} \rightarrow K^{ \pm} K^{+} K^{-}$decays. Phys. Rev. Lett. 111, 101801 (2013).

12. Aaij, R. et al. First observation of $C P$ violation in the decays of $B_{s}^{0}$ mesons. Phys. Rev. Lett. 110, 221601 (2013).

13. Hsiao, Y. K. \& Geng, C. Q. Direct $C P$ violation in $\Lambda_{b}^{0}$ decays. Phys. Rev. D 91, 116007 (2015).

14. Bensalem, W. \& London, D. T-odd triple-product correlations in hadronic $b$ decays. Phys. Rev. D 64, 116003 (2001).

15. Bensalem, W., Datta, A. \& London, D. New physics effects on triple-product correlations in $\Lambda_{b}^{0}$ decays. Phys. Rev. D 66, 094004 (2002).

16. Bensalem, W., Datta, A. \& London, D. T-violating triple-product correlations in charmless $\Lambda_{b}^{0}$ decays. Phys. Lett. B 538, 309-320 (2002).

17. Sachs, R. G. The Physics of Time Reversal (Univ. Chicago Press, 1987).

18. Branco, G. C., Lavoura, L. \& Silva, J. P. CP Violation (Oxford Univ. Press, 1999).

19. Durieux, G. \& Grossman, Y. Probing $C P$ violation systematically in differential distributions. Phys. Rev. D 92, 076013 (2015).

20. Durieux, G. CP violation in multibody decays of beauty baryons. JHEP 10, 005 (2016).

21. Gasiorowicz, S. Elementary particle physics (Wiley, 1966).

22. Bigi, I. I. Y. Charm Physics: Like Botticelli in the Sistine Chapel. Preprint at https://arxiv.org/abs/hep-ph/0107102 (2001).

23. Gronau, M. \& Rosner, J. L. Triple product asymmetries in $K, D_{(s)}$ and $B_{(s)}$ decays. Phys. Rev. D 84, 096013 (2011).

24. Gronau, M. \& Rosner, J. L. Triple product asymmmetries in $\Lambda_{b}^{0}$ and $\Xi_{b}^{0}$ decays. Phys. Lett. B 749, 104-107 (2015).

25. Valencia, G. Angular correlations in the decay $B \rightarrow V V$ and $C P$ violation. Phys. Rev. D 39, 3339-3345 (1989).

26. Aaij, R. et al. Search for $C P$ violation using $T$-odd correlations in $D^{0} \rightarrow K^{+} K^{-} \pi^{+} \pi^{-}$decays. JHEP 10, 005 (2014).

27. Aaij, R. et al. Measurement of $b$-hadron production fractions in $7 \mathrm{TeV} p p$ collisions. Phys. Rev. D 85, 032008 (2012). 
28. Aaij, R. et al. Study of the kinematic dependences of $\Lambda_{b}^{0}$ production in $p p$ collisions and a measurement of the $\Lambda_{b}^{0} \rightarrow \Lambda_{c}^{+} \pi^{-}$branching fraction. JHEP 08, 143 (2014).

29. Alves, A. A. Jr et al. The LHCb detector at the LHC. JINST 3, S08005 (2008).

30. Aaij, R. et al. LHCb detector performance. Int. J. Mod. Phys. A 30, 1530022 (2015)

31. LHCb collaboration. LHC-B: A Dedicated LHC Collider Beauty Experiment for Precision Measurements of CP Violation Letter of intent (CERN-LHCC-95-05, 1995); http://cds.cern.ch/record/290868

32. Sjöstrand, T., Mrenna, S. \& Skands, P. PYTHIA 6.4 physics and manual. JHEP 05, 026 (2006).

33. Sjöstrand, T., Mrenna, S. \& Skands, P. A brief introduction to PYTHIA 8.1. Comput. Phys. Commun. 178, 852-867 (2008).

34. Belyaev, I. et al. Handling of the generation of primary events in Gauss, the LHCb simulation framework. J. Phys. Conf. Ser. 331, 032047 (2011).

35. Lange, D. J. The EvtGen particle decay simulation package. Nucl. Instrum. Meth. A 462, 152-155 (2001).

36. Allison, J. et al. Geant4 developments and applications. IEEE Trans. Nucl. Sci. 53, 270-278 (2006).

37. Agostinelli, S. et al. Geant4: A simulation toolkit. Nucl. Instrum. Meth. A 506, 250-303 (2003).

38. Clemencic, M. et al. The LHCb simulation application, Gauss: design, evolution and experience. J. Phys. Conf. Ser. 331, 032023 (2011).

39. Olive, K. A. et al. Review of particle physics. Chin. Phys. C 38, 090001 (2014)

40. Breiman, L., Friedman, J. H., Olshen, R. A. \& Stone, C. J. Classification and Regression Trees (Wadsworth International Group, 1984).

41. Pivk, M. \& Le Diberder, F. R. sPlot: a statistical tool to unfold data distributions. Nucl. Instrum. Meth. A 555, 356-369 (2005).

42. Skwarnicki, T. A study of the radiative CASCADE transitions between the Upsilon-Prime and Upsilon resonances (Ph.D. thesis, 1986); https://inspirehep.net/record/230779

43. Albrecht, H. et al. Search for hadronic $b \rightarrow u$ decays. Phys. Lett. B 241, 278-282 (1990).

44. Fisher, R. A. The design of experiments (Oliver and Boyd Ltd., 1935).

45. Data analysis in high energy physics (eds Behnke, O., Kröninger, K., Schörner-Sadenius, T. \& Schott, G.) (Wiley, 2013).

\section{Acknowledgements}

We express our gratitude to our colleagues in the CERN accelerator departments for the excellent performance of the LHC. We thank the technical and administrative staff at the
LHCb institutes. We acknowledge support from CERN and from the national agencies: CAPES, CNPq, FAPERJ and FINEP (Brazil); NSFC (China); CNRS/IN2P3 (France); BMBF, DFG and MPG (Germany); INFN (Italy); FOM and NWO (The Netherlands); MNiSW and NCN (Poland); MEN/IFA (Romania); MinES and FASO (Russia); MinECo (Spain); SNSF and SER (Switzerland); NASU (Ukraine); STFC (United Kingdom); NSF (USA). We acknowledge the computing resources that are provided by CERN, IN2P3 (France), KIT and DESY (Germany), INFN (Italy), SURF (The Netherlands), PIC (Spain), GridPP (United Kingdom), RRCKI and Yandex LLC (Russia), CSCS (Switzerland), IFIN-HH (Romania), CBPF (Brazil), PL-GRID (Poland) and OSC (USA). We are indebted to the communities behind the multiple open source software packages on which we depend. Individual groups or members have received support from $\mathrm{AvH}$ Foundation (Germany), EPLANET, Marie Skłodowska-Curie Actions and ERC

(European Union), Conseil Général de Haute-Savoie, Labex ENIGMASS and OCEVU, Région Auvergne (France), RFBR and Yandex LLC (Russia), GVA, XuntaGal and GENCAT (Spain), Herchel Smith Fund, The Royal Society, Royal Commission for the Exhibition of 1851 and the Leverhulme Trust (United Kingdom).

\section{Author contributions}

All authors have contributed to the publication, being variously involved in the design and the construction of the detectors, in writing software, calibrating sub-systems, operating the detectors and acquiring data, and finally analysing the processed data

\section{Additional information}

Reprints and permissions information is available online at www.nature.com/reprints Correspondence and requests for materials should be addressed to

N. Neri (nicola.neri@mi.infn.it).

\section{Competing financial interests}

The authors declare no competing financial interests.

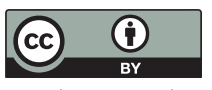

This article is licensed under a Creative Commons Attribution 4.0 International License, which permits use, sharing, adaptation, distribution and reproduction in any medium or format, as long as you give appropriate credit to the original author(s) and the source, provide a link to the Creative Commons license, and indicate if changes were made.

The images or other third party material in this article are included in the article's Creative Commons license, unless indicated otherwise in a credit line to the material. If material is not included in the article's Creative Commons license and your intended use is not permitted by statutory regulation or exceeds the permitted use, you will need to obtain permission directly from the copyright holder. To view a copy of this license, visit http://creativecommons.org/licenses/by/4.0/. 


\section{The LHCb collaboration}

R. Aaij ${ }^{40}$, B. Adeva ${ }^{39}$, M. Adinolfi ${ }^{48}$, Z. Ajaltouni ${ }^{5}$, S. Akar ${ }^{6}$, J. Albrecht ${ }^{10}$, F. Alessio $^{40}$, M. Alexander ${ }^{53}$, S. Ali ${ }^{43}$, G. Alkhazov $^{31}$, P. Alvarez Cartelle ${ }^{55}$, A. A. Alves Jr ${ }^{59}$, S. Amato 2 , S. Amerio ${ }^{23}$, Y. Amhis ${ }^{7}$, L. An ${ }^{41}$, L. Anderlini ${ }^{18}$, G. Andreassii ${ }^{41}$, M. Andreotti ${ }^{17,8}$, J. E. Andrews ${ }^{60}$, R. B. Appleby ${ }^{56}$, F. Archilli ${ }^{43}$, P. d'Argent ${ }^{12}$, J. Arnau Romeu ${ }^{6}$, A. Artamonov ${ }^{37}$, M. Artuso $^{61}$, E. Aslanides ${ }^{6}$, G. Auriemma ${ }^{26}$, M. Baalouch ${ }^{5}$, I. Babuschkin ${ }^{56}$, S. Bachmannn' ${ }^{12}$, J. J. Back ${ }^{50}$, A. Badalov ${ }^{38}$, C. Baesso ${ }^{62}$, S. Baker ${ }^{55}$, W. Baldini" ${ }^{17}$, R. J. Barlow ${ }^{56}$, C. Barschel ${ }^{40}$, S. Barsuk, W. Barter ${ }^{40}$, M. Baszczyk ${ }^{27}$, V. Batozskaya ${ }^{29}$, B. Batsukh ${ }^{61}$, V. Battista ${ }^{41}$, A. Bay ${ }^{41}$, L. Beaucourt ${ }^{4}$, J. Beddow ${ }^{53}$, F. Bedeschi ${ }^{24}$, I. Bediaga', L. J. Bel ${ }^{43}$, V. Bellee ${ }^{41}$, N. Belloli 1,i, K. Belous ${ }^{37}$, I. Belyaev ${ }^{32}$, E. Ben-Haim ${ }^{8}$, G. Bencivenni' ${ }^{19}$, S. Benson ${ }^{43}$, J. Benton ${ }^{48}$, A. Berezhnoy ${ }^{33}$, R. Bernet ${ }^{42}$, A. Bertolin ${ }^{23}$, F. Betti ${ }^{15}$, M. $-O$. Bettler ${ }^{40}$, M. van Beuzekom ${ }^{43}$, I. Bezshyiko ${ }^{42}$, S. Bifani ${ }^{47}$, P. Billoir ${ }^{8}$, T. Bird ${ }^{56}$, A. Birnkraut ${ }^{10}$, A. Bitadze ${ }^{56}$, A. Bizzeti ${ }^{18,4}$, T. Blake ${ }^{50,}$ F. Blanc ${ }^{41}$, J. Blouw ${ }^{11}$, S. Blusk ${ }^{61}$, V. Bocci ${ }^{26}$, T. Boettcher ${ }^{58}$, A. Bondar ${ }^{36}$, N. Bondar ${ }^{31,40}$, W. Bonivento ${ }^{16}$, A. Borgheresi ${ }^{21, i}$, S. Borghi ${ }^{56}$, M. Borisyak ${ }^{35}$, M. Borsato ${ }^{39}$, F. Bossu7 7 , M. Boubdir 9 , T. J. V. Bowcock ${ }^{54}$, E. Bowen ${ }^{42}$, C. Bozzi17,40, S. Braun ${ }^{12}$, M. Britsch'12, T. Britton ${ }^{61}$, J. Brodzicka ${ }^{56}$, E. Buchanann ${ }^{48}$, C. Burr ${ }^{56}$, A. Bursche ${ }^{2}$, J. Buytaert ${ }^{40}$, S. Cadeddu ${ }^{16}$, R. Calabrese ${ }^{17,8}$, M. Calvi ${ }^{21, i}$, M. Calvo Gomez ${ }^{38, m}$, A. Camboni ${ }^{38}$, P. Campana ${ }^{19}$, D. Campora Perez ${ }^{40}$, D. H. Campora Perez ${ }^{40}$, L. Capriotti ${ }^{56}$, A. Carbone ${ }^{15, \mathrm{e}}$, G. Carboni ${ }^{25, j}$, R. Cardinale ${ }^{20, h}$, A. Cardini ${ }^{16}$, P. Carniti21,i, L. Carson ${ }^{52}$, K. Carvalho Akiba $^{2}$, G. Casse ${ }^{54}$, L. Cassina 21,i, L. Castillo Garcia ${ }^{41}$, M. Cattaneo ${ }^{40}$, Ch. Cauet $^{10}$, G. Cavallero ${ }^{20}$, R. Cenci ${ }^{24, t}$, M. Charles ${ }^{8}$, Ph. Charpentier ${ }^{40}$, G. Chatzikonstantinidis ${ }^{47}$, M. Chefdeville ${ }^{4}$, S. Chen ${ }^{56}$, S.-F. Cheung ${ }^{57}$, V. Chobanova ${ }^{39}$, M. Chrzaszcz ${ }^{42,27}$, X. Cid Vidal $^{39}$,

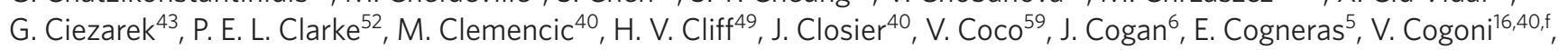
L. Cojocariu ${ }^{30}$, G. Collazuol ${ }^{23,0}$, P. Collins ${ }^{40}$, A. Comerma-Montells ${ }^{12}$, A. Contu ${ }^{40}$, A. Cook ${ }^{48}$, S. Coquereau ${ }^{38}$, G. Corti $^{40}$, M. Corvo ${ }^{17,8}$, C. M. Costa Sobral ${ }^{50}$, B. Couturier ${ }^{40}$, G. A. Cowan ${ }^{52}$, D. C. Craik ${ }^{52}$, A. Crocombe ${ }^{50}$, M. Cruz Torres ${ }^{62}$, S. Cunliffe ${ }^{55}$, R. Currie ${ }^{55}$, C. D'Ambrosio ${ }^{40}$, F. Da Cunha Marinho², E. Dall'Occo ${ }^{43}$, J. Dalseno ${ }^{48}$, P. N. Y. David ${ }^{43}$, A. Davis ${ }^{59}$, O. De

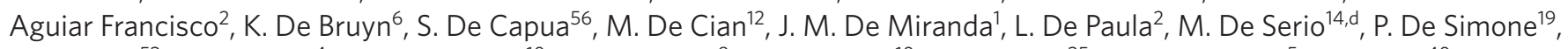
C.-T. Dean ${ }^{53}$, D. Decamp ${ }^{4}$, M. Deckenhoff ${ }^{10}$, L. Del Buono ${ }^{8}$, M. Demmer ${ }^{10}$, D. Derkach ${ }^{35}$, O. Deschamps ${ }^{5}$, F. Dettori ${ }^{40}$,

B. Dey ${ }^{22}$, A. Di Canto ${ }^{40}$, H. Dijkstra ${ }^{40}$, F. Dordei ${ }^{40}$, M. Dorigo ${ }^{41}$, A. Dosil Suárez ${ }^{39}$, A. Dovbnya ${ }^{45}$, K. Dreimanis ${ }^{54}$, L. Dufour ${ }^{43}$, G. Dujany ${ }^{56}$, K. Dungs $^{40}$, P. Durante ${ }^{40}$, R. Dzhelyadin ${ }^{37}$, A. Dziurda $^{40}$, A. Dzyuba ${ }^{31}$, N. Déléage ${ }^{4}$, S. Easo ${ }^{51}$, M. Ebert ${ }^{52}$, U. Egede ${ }^{55}$, V. Egorychev ${ }^{32}$, S. Eidelman ${ }^{36}$, S. Eisenhardt ${ }^{52}$, U. Eitschberger ${ }^{10}$, R. Ekelhof ${ }^{10}$, L. Eklund ${ }^{53}$, Ch. Elsasser $^{42}$, S. Ely ${ }^{61}$, S. Esen ${ }^{12}$, H. M. Evans ${ }^{49}$, T. Evans ${ }^{57}$, A. Falabella ${ }^{15}$, N. Farley ${ }^{47}$, S. Farry ${ }^{54}$, R. Fay ${ }^{54}$, D. Fazzini ${ }^{21,}$, D. Ferguson ${ }^{52}$,

V. Fernandez Albor ${ }^{39}$, A. Fernandez Prieto ${ }^{39}$, F. Ferrari ${ }^{15,40}$, F. Ferreira Rodrigues ${ }^{1}$, M. Ferro-Luzzi ${ }^{40}$, S. Filippov ${ }^{34}$, R. A. Fini ${ }^{14}$, M. Fiore ${ }^{17,8}$, M. Fiorini ${ }^{17,8}$, M. Firlej ${ }^{28}$, C. Fitzpatrick ${ }^{41}$, T. Fiutowski ${ }^{28}$, F. Fleuret ${ }^{7, b}$, K. Fohl ${ }^{40}$, M. Fontana ${ }^{16,40}$, F. Fontanelli ${ }^{20, h}$, D. C. Forshaw ${ }^{61}$, R. Forty 40, V. Franco Lima ${ }^{54}$, M. Frank ${ }^{40}$, C. Frei ${ }^{40}$, J. Fu 22,a, E. Furfaro ${ }^{25, j}$, C. Färber ${ }^{40}$, A. Gallas Torreira ${ }^{39}$, D. Galli15,e, S. Gallorini23, S. Gambetta52, M. Gandelman², P. Gandinin7, Y. Gao ${ }^{3}$, L. M. Garcia Martin ${ }^{68}$, J. Garcıa Pardiñas ${ }^{39}$, J. Garra Tico ${ }^{49}$, L. Garrido ${ }^{38}$, P. J. Garsed ${ }^{49}$, D. Gascon ${ }^{38}$, C. Gaspar ${ }^{40}$, L. Gavardi' ${ }^{10}$, G. Gazzoni ${ }^{5}$, D. Gerick², E. Gersabeck ${ }^{12}$, M. Gersabeck ${ }^{56}$, T. Gershon ${ }^{50}$, Ph. Ghez ${ }^{4}$, S. Gianì ${ }^{41}$, V. Gibson ${ }^{49}$, O. G. Girard ${ }^{41}$, L. Giubega ${ }^{30}$, K. Gizdov², V. V. Gligorov ${ }^{8}$, D. Golubkov ${ }^{32}$, A. Golutvin 55,40 , A. Gomes ${ }^{1, a}$, I. V. Gorelov ${ }^{33}$, C. Gotti 11, , M. Grabalosa Gándara ${ }^{5}$, R. Graciani Diaz ${ }^{38}$,

L. A. Granado Cardoso ${ }^{40}$, E. Graugés ${ }^{38}$, E. Graverini ${ }^{42}$, G. Graziani ${ }^{18}$, A. Grecu ${ }^{30}$, P. Griffith ${ }^{47}$, L. Grillo ${ }^{21,40, i}$,

B. R. Gruberg Cazon ${ }^{57}$, O. Grünberg ${ }^{66}$, E. Gushchin ${ }^{34}$, Yu. Guz ${ }^{37}$, T. Gys ${ }^{40}$, C. Göbel $^{62}$, T. Hadavizadeh ${ }^{57}$, C. Hadjivasiliou ${ }^{5}$, G. Haefeli41 ${ }^{41}$ C. Haen ${ }^{40}$, S. C. Haines ${ }^{49}$, S. Hall ${ }^{55}$, B. Hamilton ${ }^{60}$, X. Hann ${ }^{12}$, S. Hansmann-Menzemer ${ }^{12}$, N. Harnew ${ }^{57}$, S. T. Harnew ${ }^{48}$, J. Harrison ${ }^{56}$, M. Hatch ${ }^{40}$, J. He ${ }^{63}$, T. Head ${ }^{41}$, A. Heister ${ }^{9}$, K. Hennessy ${ }^{54}$, P. Henrard ${ }^{5}$, L. Henry ${ }^{8}$, J. A. Hernando Morata ${ }^{39}$, E. van Herwijnen ${ }^{40}$, M. Heß $^{66}$, A. Hicheur ${ }^{2}$, D. Hill ${ }^{57}$, C. Hombach ${ }^{56}$, H. Hopchev ${ }^{41}$, W. Hulsbergen ${ }^{43}$, T. Humair ${ }^{55}$, M. Hushchyn ${ }^{35}$, N. Hussain ${ }^{57}$, D. Hutchcroft ${ }^{54}$, M. Idzik ${ }^{28}$, P. Ilten ${ }^{58}$, R. Jacobsson ${ }^{40}$, A. Jaeger ${ }^{12}$, J. Jalocha ${ }^{57}$, E. Jans ${ }^{43}$, A. Jawahery ${ }^{60}$, F. Jiang ${ }^{3}$, M. John ${ }^{57}$, D. Johnson ${ }^{40}$, C. R. Jones ${ }^{49}$, C. Joram ${ }^{40}$, B. Jost ${ }^{40}$, N. Jurik ${ }^{61}$, S. Kandybei ${ }^{45}$, W. Kanso ${ }^{6}$, M. Karacson ${ }^{40}$, J. M. Kariuki ${ }^{48}$, S. Karodia ${ }^{53}$, M. Kecke ${ }^{12}$, M. Kelsey ${ }^{61}$, I. R. Kenyon ${ }^{47}$, M. Kenzie ${ }^{49}$, T. Ketel ${ }^{44}$,

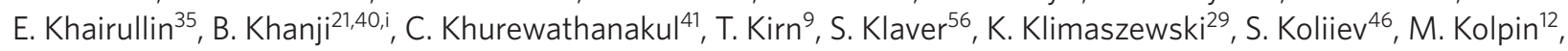
I. Komarov ${ }^{41}$, R. F. Koopman ${ }^{44}$, P. Koppenburg ${ }^{43}$, A. Kozachuk ${ }^{33}$, M. Kozeiha ${ }^{5}$, L. Kravchuk ${ }^{34}, K^{2}$ Kreplin ${ }^{12}$, M. Kreps ${ }^{50}$, P. Krokovny ${ }^{36}$, F. Kruse ${ }^{10}$, W. Krzemien ${ }^{29}$, W. Kucewicz ${ }^{27, I}$, M. Kucharczyk ${ }^{27}$, V. Kudryavtsev ${ }^{36}$, A. K. Kuonen ${ }^{41}$, K. Kurek ${ }^{29}$, T. Kvaratskheliya ${ }^{32,40}$, D. Lacarrere ${ }^{40}$, G. Lafferty ${ }^{56}$, A. Lai ${ }^{16}$, D. Lambert ${ }^{52}$, G. Lanfranchi ${ }^{19}$, C. Langenbruch ${ }^{9}$, T. Latham ${ }^{50}$,

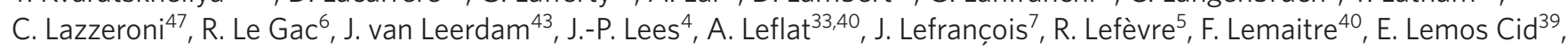

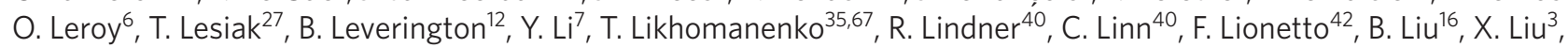
D. Loh ${ }^{50}$, I. Longstaff ${ }^{53}$, J. H. Lopes ${ }^{2}$, D. Lucchesi ${ }^{23,0}$, M. Lucio Martinez ${ }^{39}$, H. Luo ${ }^{52}$, A. Lupato ${ }^{23}$, E. Luppi17, , O. Lupton ${ }^{57}$,

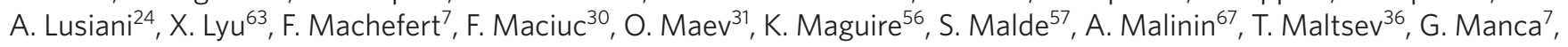
G. Mancinelli, , P. Manning ${ }^{61}$, J. Maratas ${ }^{5, v}$, J. F. Marchand ${ }^{4}$, U. Marconi ${ }^{15}$, C. Marin Benito ${ }^{38}$, P. Marino ${ }^{24, t}$, J. Marks ${ }^{12}$, G. Martellotti ${ }^{26}$, M. Martin ${ }^{6}$, M. Martinelli ${ }^{41}$, D. Martinez Santos ${ }^{39}$, F. Martinez Vidal ${ }^{68}$, D. Martins Tostes ${ }^{2}$, L. M. Massacrier ${ }^{7}$, A. Massafferri', R. Matev 40, A. Mathad ${ }^{50}$, Z. Mathe ${ }^{40}$, C. Matteuzzi ${ }^{21}$, A. Mauri42, B. Maurin ${ }^{41}$, A. Mazurov ${ }^{47}$, M. McCann ${ }^{55}$, J. McCarthy ${ }^{47}, \mathrm{~A} . \mathrm{McNab}^{56}$, R. McNulty ${ }^{13}$, B. Meadows ${ }^{59}$, F. Meier $^{10}$, M. Meissner ${ }^{12}$, D. Melnychuk ${ }^{29}$, M. Merk ${ }^{43}$, A. Merli ${ }^{22,9}$, E. Michielin"23, D. A. Milanes ${ }^{65}$, M.-N. Minard ${ }^{4}$, D. S. Mitzel12, A. Mogini ${ }^{8}$, J. Molina Rodriguez ${ }^{62}$, I. A. Monroy ${ }^{65}$, S. Monteil', M. Morandin ${ }^{23}$, P. Morawski28, A. Mordà 6 , M. J. Morello ${ }^{24, t}$, J. Moron ${ }^{28}$, A. B. Morris ${ }^{52}$, R. Mountain ${ }^{61}$, F. Muheim ${ }^{52}$, M. Mulder ${ }^{43}$, M. Mussini ${ }^{15}$, D. Müller ${ }^{56}$, J. Müller ${ }^{10}$, K. Müller $^{42}$, V. Müller $^{10}$, P. Naik ${ }^{48}$, T. Nakada ${ }^{41}$, R. Nandakumar ${ }^{51}$, A. Nandi ${ }^{57}$, I. Nasteva ${ }^{2}$, M. Needham ${ }^{52}$, N. Neri' ${ }^{22}$, S. Neubert ${ }^{12}$, N. Neufeld ${ }^{40}$, M. Neuner ${ }^{12}$, A. D. Nguyen ${ }^{41}$, C. Nguyen-Mau ${ }^{41, n}$, 
S. Nieswand ${ }^{9}$, R. Niet ${ }^{10}$, N. Nikitin ${ }^{33}$, T. Nikodem ${ }^{12}$, A. Novoselov ${ }^{37}$, D. P. O'Hanlon ${ }^{50}$, A. Oblakowska-Mucha ${ }^{28}$, V. Obraztsov ${ }^{37}$, S. Ogilvy ${ }^{19}$, R. Oldeman ${ }^{49}$, C. J. G. Onderwater ${ }^{69}$, J. M. Otalora Goicochea ${ }^{2}$, A. Otto ${ }^{40}$, P. Owen ${ }^{42}$, A. Oyanguren ${ }^{68}$, P. R. Pais ${ }^{41}$, A. Palano ${ }^{14, \mathrm{~d}}$, F. Palombo $22, \mathrm{a}$, M. Palutan ${ }^{19}$, J. Panman ${ }^{40}$, A. Papanestis ${ }^{51}$, M. Pappagallo ${ }^{14, \mathrm{~d}}$, L. L. Pappalardo ${ }^{17,8}$, W. Parker ${ }^{60}$ C. Parkes ${ }^{56}$, G. Passaleva ${ }^{18}$, A. Pastore ${ }^{14, d}$, G. D. Patel ${ }^{54}$, M. Pate $^{55}$, C. Patrignani ${ }^{15, e}$, A. Pearce ${ }^{56,51}$, A. Pellegrino ${ }^{43}$, G. Penso ${ }^{26}$, M. Pepe Altarelli ${ }^{40}$, S. Perazzini ${ }^{40}$, P. Perret ${ }^{5}$, L. Pescatore ${ }^{47}$, K. Petridis ${ }^{48}$, A. Petrolini ${ }^{20, h}$, A. Petrov ${ }^{67}$, M. Petruzzo ${ }^{22,9}$, E. Picatoste Olloqui ${ }^{38}$, B. Pietrzyk ${ }^{4}$, M. Pikies ${ }^{27}$, D. Pinci ${ }^{26}$, A. Pistone ${ }^{20}$, A. Piucci ${ }^{12}$, S. Playfer ${ }^{52}$, M. Plo Casasus ${ }^{39}$, T. Poikela ${ }^{40}$, F. Polci ${ }^{8}$, A. Poluektov 50,36, I. Polyakov ${ }^{61}$, E. Polycarpo², G. J. Pomery ${ }^{48}$, A. Popov ${ }^{37}$, D. Popov ${ }^{11,40}$, B. Popovici ${ }^{30}$, S. Poslavskii ${ }^{37}$, C. Potterat ${ }^{2}$, E. Price ${ }^{48}$, J. D. Price ${ }^{54}$, J. Prisciandaro ${ }^{39}$, A. Pritchard ${ }^{54}$, C. Prouve ${ }^{48}$, V. Pugatch ${ }^{46}$, A. Puig Navarro ${ }^{41}$, G. Punzi ${ }^{24, p}$ W. Qian ${ }^{57}$, R. Quagliani 7,48, B. Rachwal27, J. H. Rademacker ${ }^{48}$, M. Rama ${ }^{24}$, M. Ramos Pernas ${ }^{39}$, M. S. Rangel ${ }^{2}$, I. Raniuk ${ }^{45}$, G. Raven ${ }^{44}$, F. Redi ${ }^{55}$, S. Reichert ${ }^{10}$, A. C. dos Reis ${ }^{1}$, C. Remon Alepuz ${ }^{68}$, V. Renaudin ${ }^{7}$, S. Ricciardi ${ }^{51}$, S. Richards ${ }^{48}$, M. Rihl ${ }^{40}$, K. Rinnert ${ }^{54}$, V. Rives Molina ${ }^{38}$, P. Robbe ${ }^{7,40}$, A. B. Rodrigues ${ }^{1}$, E. Rodrigues ${ }^{59}$, J. A. Rodriguez Lopez ${ }^{65}$, P. Rodriguez Perez ${ }^{56}$, A. Rogozhnikov ${ }^{35}$, S. Roiser ${ }^{40}$, V. Romanovskiy ${ }^{37}$, A. Romero Vidal ${ }^{39}$, J. W. Ronayne ${ }^{13}$, M. Rotondo ${ }^{19}$, M. S. Rudolph ${ }^{61}$, T. Ruf ${ }^{40}$, P. Ruiz Valls ${ }^{68}$, J. J. Saborido Silva ${ }^{39}$, E. Sadykhov ${ }^{32}$, N. Sagidova ${ }^{31}$, B. Saitta ${ }^{16, f}$, V. Salustino Guimaraes ${ }^{2}$, C. Sanchez Mayordomo ${ }^{68}$, B. Sanmartin Sedes ${ }^{39}$, R. Santacesaria ${ }^{26}$, C. Santamarina Rios ${ }^{39}$, M. Santimaria ${ }^{19}$, E. Santovetti ${ }^{25, j}$, A. Sarti ${ }^{19, k}$, C. Satriano ${ }^{26,5}$, A. Satta ${ }^{25}$, D. M. Saunders ${ }^{48}$, D. Savrina ${ }^{32,33}$, S. Schael ${ }^{9}$, M. Schellenberg ${ }^{10}$, M. Schiller ${ }^{40}$, H. Schindler ${ }^{40}$, M. Schlupp ${ }^{10}$, M. Schmelling ${ }^{11}$, T. Schmelzer ${ }^{10}$, B. Schmidt ${ }^{40}$, O. Schneider ${ }^{41}$, A. Schopper ${ }^{40}$, K. Schubert $^{10}$, M. Schubiger ${ }^{41}$, M.-H. Schune ${ }^{7}$, R. Schwemmer ${ }^{40}$, B. Sciascia ${ }^{19}$, A. Sciubba ${ }^{26, k}$, A. Semennikov ${ }^{32}$, A. Sergi ${ }^{47}$, N. Serra ${ }^{42}$, J. Serrano ${ }^{6}$, L. Sestini ${ }^{23}$, P. Seyfert ${ }^{21}$, M. Shapkin ${ }^{37}$, I. Shapoval ${ }^{45}$, Y. Shcheglov' ${ }^{31}$, T. Shears ${ }^{54}$, L. Shekhtman ${ }^{36}$, V. Shevchenko ${ }^{67}$, A. Shires ${ }^{10}$, B. G. Siddi ${ }^{17,40}$, R. Silva Coutinho ${ }^{42}$, L. Silva de Oliveira ${ }^{2}$, G. Simi ${ }^{23,0}$, S. Simone ${ }^{14, d}$, M. Sirendi ${ }^{49}$, N. Skidmore ${ }^{48}$, T. Skwarnicki ${ }^{61}$, E. Smith ${ }^{55}$, I. T. Smith ${ }^{52}$, J. Smith ${ }^{49}$, M. Smith ${ }^{55}$, H. Snoek ${ }^{43}$, M. D. Sokoloff ${ }^{59}$, F. J. P. Soler ${ }^{53}$, B. Souza De Paula², B. Spaan ${ }^{10}$, P. Spradlin ${ }^{53}$, S. Sridharan ${ }^{40}$, F. Stagni ${ }^{40}$, M. Stahl ${ }^{12}$, S. Stahl ${ }^{40}$, P. Stefko ${ }^{41}$, S. Stefkova ${ }^{55}$, O. Steinkamp ${ }^{42}$,

S. Stemmle ${ }^{12}$, O. Stenyakin ${ }^{37}$, S. Stevenson ${ }^{57}$, S. Stoica ${ }^{30}$, S. Stone ${ }^{61}$, B. Storaci ${ }^{42}$, S. Stracka ${ }^{24, \text {, }}$, M. Straticiuc ${ }^{30}$, U. Straumann ${ }^{42}$, L. Sun ${ }^{59}$, W. Sutcliffe ${ }^{55}$, K. Swientek ${ }^{28}$, V. Syropoulos ${ }^{44}$, M. Szczekowski ${ }^{29}$, T. Szumlak ${ }^{28}$, S. T'Jampens ${ }^{4}$, A. Tayduganov ${ }^{6}$, T. Tekampe ${ }^{10}$, G. Tellarini ${ }^{17,8}$, F. Teubert ${ }^{40}$, E. Thomas ${ }^{40}$, J. van Tilburg ${ }^{43}$, M. J. Tilley ${ }^{55}$, V. Tisserand ${ }^{4}$, M. Tobin ${ }^{41}$, S. Tolk ${ }^{49}$, L. Tomassetti17,8, D. Tonelli40 ${ }^{40}$, S. Topp-Joergensen ${ }^{57}$, F. Toriello61, E. Tournefier ${ }^{4}$, S. Tourneur ${ }^{41}$, K. Trabelsi ${ }^{41}$, M. Traill ${ }^{53}$, M. T. $\operatorname{Tran}^{41}$, M. Tresch ${ }^{42}$, A. Trisovic ${ }^{40}$, A. Tsaregorodtsev6 ${ }^{6}$ P. Tsopelas ${ }^{43}$, A. Tully ${ }^{49}$, N. Tuning ${ }^{43}$, A. Ukleja ${ }^{29}$, A. Ustyuzhanin ${ }^{35,67}$, U. Uwer ${ }^{12}$, C. Vacca ${ }^{16, f}$, V. Vagnoni ${ }^{15,40}$, A. Valassi ${ }^{40}$, S. Valat ${ }^{40}$, G. Valenti15, A. Vallier ${ }^{7}$, R. Vazquez Gomez ${ }^{19}$, P. Vazquez Regueiro ${ }^{39}$, S. Vecchi ${ }^{17}$, M. van Veghel ${ }^{43}$, J. J. Velthuis ${ }^{48}$, M. Veltri ${ }^{18, r}$, G. Veneziano ${ }^{41}$, A. Venkateswaran ${ }^{61}$, M. Vernet ${ }^{5}$, M. Vesterinen ${ }^{12}$, B. Viaud ${ }^{7}$, D. Vieira', M. Vieites Diaz ${ }^{39}$, X. Vilasis-Cardona $38, m$, V. Volkov ${ }^{33}$, A. Vollhardt ${ }^{42}$, B. Voneki ${ }^{40}$, A. Vorobyev ${ }^{31}$, V. Vorobyev ${ }^{36}$, C. Voß ${ }^{66}$, J. A. de Vries ${ }^{43}$, C. Vázquez Sierra ${ }^{39}$, R. Waldi ${ }^{66}$, C. Wallace ${ }^{50}$, R. Wallace ${ }^{13}$, J. Walsh ${ }^{24}$, J. Wang ${ }^{61}$, D. R. Ward ${ }^{49}$, H. M. Wark ${ }^{54}$, N. K. Watson ${ }^{47}$, D. Websdale ${ }^{55}$, A. Weiden ${ }^{42}$, M. Whitehead ${ }^{40}$, J. Wicht ${ }^{50}$, G. Wilkinson ${ }^{57,40}$, M. Wilkinson ${ }^{61}$, M. Williams ${ }^{40}$, M. P. Williams ${ }^{47}$, M. Williams ${ }^{58}$, T. Williams ${ }^{47}$, F. F. Wilson ${ }^{51}$, J. Wimberley ${ }^{60}$, J. Wishahi ${ }^{10}$, W. Wislicki ${ }^{29}$, M. Witek ${ }^{27}$, G. Wormser ${ }^{7}$, S. A. Wotton ${ }^{49}$, K. Wraight ${ }^{53}$, S. Wright ${ }^{49}$, K. Wyllie ${ }^{40}, Y . X_{i e^{64}}$, Z. Xing ${ }^{61}$, Z. Xu ${ }^{41}$, Z. Yang ${ }^{3}$, H. Yin ${ }^{64}$, J. Yu ${ }^{64}$, X. Yuan ${ }^{36}$, O. Yushchenko ${ }^{37}$, K. A. Zarebski ${ }^{47}$,

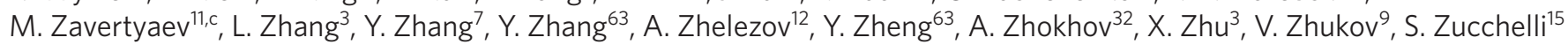

\section{Primary affiliations}

${ }^{1}$ Centro Brasileiro de Pesquisas Físicas (CBPF), Rio de Janeiro 22290-180, Brazil. ${ }^{2}$ Universidade Federal do Rio de Janeiro (UFRJ), Rio de Janeiro 21941-972, Brazil. ${ }^{3}$ Center for High Energy Physics, Tsinghua University, Beijing 100084, China. ${ }^{4}$ LAPP, Université Savoie Mont-Blanc, CNRS/IN2P3,

Annecy-Le-Vieux 74941, France. ${ }^{5}$ Clermont Université, Université Blaise Pascal, CNRS/IN2P3, LPC, Clermont-Ferrand 63177, France. ${ }^{6}$ CPPM, Aix-Marseille Université, CNRS/IN2P3, Marseille 12388, France. ${ }^{7}$ LAL, Université Paris-Sud, CNRS/IN2P3, Orsay 91898, France. ${ }^{8}$ LPNHE, Université Pierre et Marie Curie, Université Paris Diderot, CNRS/IN2P3, Paris 75252, France. ${ }^{9}$ I. Physikalisches Institut, RWTH Aachen University, Aachen D-52056, Germany.

${ }^{10}$ Fakultät Physik, Technische Universität Dortmund, Dortmund D-44221, Germany. ${ }^{11}$ Max-Planck-Institut für Kernphysik (MPIK), Heidelberg D-69029, Germany. ${ }^{12}$ Physikalisches Institut, Ruprecht-Karls-Universität Heidelberg, Heidelberg D-69120, Germany. ${ }^{13}$ School of Physics, University College Dublin, Dublin 4, Ireland. ${ }^{14}$ Sezione INFN di Bari, Bari I-70126, Italy. ${ }^{15}$ Sezione INFN di Bologna, Bologna I-40126, Italy. ${ }^{16}$ Sezione INFN di Cagliari, Cagliari I-09042, Italy. ${ }^{17}$ Sezione INFN di Ferrara, Ferrara I-44100, Italy. ${ }^{18}$ Sezione INFN di Firenze, Firenze I-50019, Italy. ${ }^{19}$ Laboratori Nazionali dell'INFN di Frascati, Frascati I-00044, Italy. ${ }^{20}$ Sezione INFN di Genova, Genova I-16146, Italy. ${ }^{21}$ Sezione INFN di Milano Bicocca, Milano I-20133, Italy. ${ }^{22}$ Sezione INFN di Milano, Milano I-20133, Italy. ${ }^{23}$ Sezione INFN di Padova, Padova I-35131, Italy. ${ }^{24}$ Sezione INFN di Pisa, Pisa I-56127, Italy. ${ }^{25}$ Sezione INFN di Roma Tor Vergata, Roma I-00133, Italy. ${ }^{26}$ Sezione INFN di Roma La Sapienza, Roma I-00185, Italy. ${ }^{27}$ Henryk Niewodniczanski Institute of Nuclear Physics Polish Academy of Sciences, Kraków PL-31-342, Poland. ${ }^{28}$ AGH - University of Science and Technology, Faculty of Physics and Applied Computer Science, Kraków PL-30-059, Poland. ${ }^{29}$ National Center for Nuclear Research (NCBJ), Warsaw PL- 00-681, Poland. ${ }^{30}$ Horia Hulubei National Institute of Physics and Nuclear Engineering, Bucharest-Magurele 76900, Romania. ${ }^{31}$ Petersburg Nuclear Physics Institute (PNPI), Gatchina RU-188350, Russia. ${ }^{32}$ Institute of Theoretical and Experimental Physics (ITEP), Moscow RU-117259, Russia. ${ }^{33}$ Institute of Nuclear Physics, Moscow State University (SINP MSU), Moscow 119991, Russia. ${ }^{34}$ Institute for Nuclear Research of the Russian Academy of Sciences (INR RAN), Moscow RU-117312, Russia. ${ }^{35}$ Yandex School of Data Analysis, Moscow 119021, Russia. ${ }^{36}$ Budker Institute of Nuclear Physics (SB RAS) and Novosibirsk State University, Novosibirsk RU-630-090, Russia. ${ }^{37}$ Institute for High Energy Physics (IHEP), Protvino RU0142281, Russia. ${ }^{38}$ ICCUB, Universitat de Barcelona, Barcelona E-08028, Spain. ${ }^{39}$ Universidad de Santiago de Compostela, Santiago de Compostela E-15782, Spain. ${ }^{40}$ European Organization for Nuclear Research (CERN), Geneva CH-1211, Switzerland. ${ }^{41}$ Ecole Polytechnique Fédérale de Lausanne (EPFL), Lausanne CH-1015, Switzerland. ${ }^{42}$ Physik-Institut, Universität Zürich, Zürich CH-8057, Switzerland. ${ }^{43}$ Nikhef National Institute for Subatomic Physics, Amsterdam NL-1009, The Netherlands. ${ }^{44}$ Nikhef National Institute for Subatomic Physics and VU University Amsterdam, Amsterdam NL-1081, The Netherlands. ${ }^{45}$ NSC Kharkiv Institute of Physics and Technology (NSC KIPT), Kharkiv 61108, Ukraine. ${ }^{46}$ Institute for Nuclear Research of the National Academy of Sciences (KINR), Kyiv 03680, Ukraine. ${ }^{47}$ University of Birmingham, Birmingham B15 2TT, UK. 
${ }^{48}$ H.H. Wills Physics Laboratory, University of Bristol, Bristol BS8 1TL, UK. ${ }^{49}$ Cavendish Laboratory, University of Cambridge, Cambridge CB3 OHE, UK. ${ }^{50}$ Department of Physics, University of Warwick, Coventry CV4 7AL, UK. ${ }^{51}$ STFC Rutherford Appleton Laboratory, Didcot OX11 0QX, UK. ${ }^{52}$ School of Physics and Astronomy, University of Edinburgh, Edinburgh EH9 3JZ, UK. ${ }^{53}$ School of Physics and Astronomy, University of Glasgow, Glasgow G12 8QQ, UK. ${ }^{54}$ Oliver Lodge Laboratory, University of Liverpool, Liverpool L69 7ZE, UK. ${ }^{55}$ Imperial College London, London SW7 2BZ, UK. ${ }^{56}$ School of Physics and Astronomy, University of Manchester, Manchester M13 9PL, UK. ${ }^{57}$ Department of Physics, University of Oxford, Oxford OX1 3RH, UK. ${ }^{58}$ Massachusetts Institute of Technology, Cambridge, Massachusetts 02139, USA. ${ }^{59}$ University of Cincinnati, Cincinnati, Ohio 45221-0011, USA. ${ }^{60}$ University of Maryland, College Park, Maryland 20742, USA. ${ }^{61}$ Syracuse University, Syracuse, New York 13244-1130, USA. ${ }^{62}$ Pontifícia Universidade Católica do Rio de Janeiro (PUC-Rio), Rio de Janeiro 22451-900, Brazil, associated to 2. ${ }^{63}$ University of Chinese Academy of Sciences, Beijing 100049, China, associated to 3.

${ }^{64}$ Institute of Particle Physics, Central China Normal University, Wuhan, Hubei 430079, China, associated to 3. ${ }^{65}$ Departamento de Fisica, Universidad Nacional de Colombia, Bogota 21716, Colombia, associated to 8. ${ }^{66}$ Institut für Physik, Universität Rostock, Rostock D-18051, Germany, associated to 12.

${ }^{67}$ National Research Centre Kurchatov Institute, Moscow 123182, Russia, associated to $32 .{ }^{68}$ Instituto de Fisica Corpuscular (IFIC), Universitat de Valencia-CSIC, Valencia E-46980, Spain, associated to 38. ${ }^{69}$ Van Swinderen Institute, University of Groningen, Groningen 9747 AG, The Netherlands, associated to 43.

\section{Secondary affiliations}

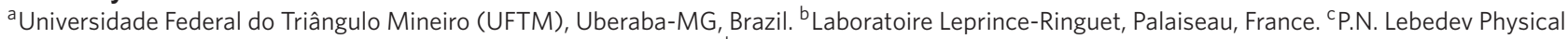
Institute, Russian Academy of Science (LPI RAS), Moscow, Russia. ' Università di Bari, Bari l-70126, Italy. ' Università di Bologna, Bologna I-40126, Italy. ${ }^{f}$ Università di Cagliari, Cagliari I-09042, Italy. '̊niversità di Ferrara, Ferrara I-44100, Italy. ' Università di Genova, Genova I-16146, Italy. 'Università di Milano Bicocca, Milano I-20133, Italy. 'Università di Roma Tor Vergata, Roma I-00133, Italy. kUniversità di Roma La Sapienza, Roma I-00185, Italy. 'AGH University of Science and Technology, Faculty of Computer Science, Electronics and Telecommunications, Kraków PL-30-059, Poland. mLIFAELS, La Salle, Universitat Ramon Llull, Barcelona, Spain. ${ }^{\mathrm{n}}$ Hanoi University of Science, Hanoi, Vietnam. ${ }^{\circ}$ Università di Padova, Padova I-35131, Italy. ${ }^{\mathrm{P} U n i v e r s i t a ̀ ~ d i ~ P i s a, ~}$ Pisa I-56127, Italy. ${ }^{9}$ Università degli Studi di Milano, Milano I-20133, Italy. 'Università di Urbino, Urbino, Italy. ${ }^{5}$ Università della Basilicata, Potenza, Italy.

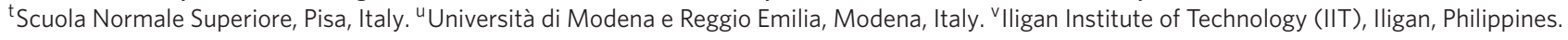

